\title{
X-DLVO Interactions between Nanocolloidal Magnetic Particles: The Quantitative Interpretation of the pH-Dependent Phase Diagram of EDL-MF
}

\author{
A. F. C. Campos, E. P. Marinho, M. de A. Ferreira, and F. A. Tourinho \\ Complex Fluids Group, Instituto de Qu'mica, Universidade de Bras'lia, \\ Caixa Postal 04478, 70919-970, Bras'lia (DF), Brazil \\ F. L. de O. Paula, J. Depeyrot \\ Complex Fluids Group, Instituto de F'sica, Universidade de Bras'lia, \\ Caixa Postal 04455, 70919-970, Bras'lia (DF), Brazil
}

(Received 01/07/2008)

\begin{abstract}
The phase behavior of acidic samples of EDL-MF based on cobalt ferrite nanoparticles with controlled mean sizes was investigated at constant temperature and in absence of magnetic field. By monitoring the nanoparticle charge by $\mathrm{pH}$ adjustments, we constructed an experimental $\mathrm{pH}$-dependent phase diagram for all samples that revealed sol, gel thixotropic or coagulated phases in different $\mathrm{pH}$ regions. Then, by using an extended DLVO potential we analyzed quantitatively the observed phase diagram in function of $\mathrm{pH}$ and nanoparticle mean size.
\end{abstract}

Keywords: EDL-MF, surface charge density, phase diagrams

\section{INTRODUCTION}

The colloidal interactions in magnetic dispersions of nanosized particles has routinely been a matter of outstanding interest in complex fluids research. In fact, because of their importance in industrial, biomedical and environmental applications, the colloidal stability of these systems must be ensured in order to prevent agglomaration phenomena. In this way, many aspects concerning magnetic colloidal behavior have currently been explored not only by experiments but also in theoretical point of view. This paper looks in a straight line to this subject.

The systems where interparticle interactions can be totally described by only van der Waals forces are rare and limited [1]. On the contrary, in electric double layered magnetic fluids (EDL-MF) there is an interplay between short-range attractive and long-range repulsive interactions which leads to a complex potential energy function. Nanoparticle interactions in EDL-MF may therefore cause colloidal instability in the primary minimum of the potential, kinetical stability in the secondary minimum or thermodynamical stability if a high enough energy barrier is present. It agrees with the experimental observations of fluid, liquid, gas-like and solid phases in these magnetic colloids [2,3,4] For instance, gasliquid-like and fluid-solid phase transitions may be evidenced varying the ionic strength or $\mathrm{pH}$ of the dispersion at constant temperature and in absence of external magnetic field [5,6]. It corresponds to monitor respectively the rate of screening in the electric double layer and the nanoparticle surface charge density. Moreover, by applying an external magnetic field or decreasing the temperature of the colloidal dispersion, such kind of phase transitions can also be observed $[7,8,9]$.

Very recently, we have investigated the surface charge density of EDL-MF based on nanoparticles with controlled mean sizes, by electrochemical measurements [10]. The results have shown that for larger particles the surface is fully ionized whereas for smaller ones the saturation value of the sur-

Electronic address: relex@unb.br face charge is strongly reduced. This behavior was enlightened considering finite size and surface effects which lead to marked changes of the coordination of surface ions. Thus, as the electrostatic repulsive component of the interparticle potential energy is strongly dependent on particle surface charge, the nanoparticle mean size seems to play an important role in the interpretation of the $\mathrm{pH}$-dependent phase diagram of EDL-MF.

In this context, the main goal of this work is to investigate the colloidal stability of EDL-MF dispersions in function of $\mathrm{pH}$ coupling experimental data with theoretical calculations. In the first part of the paper, we describe quantitatively the interparticle forces in the EDL-MF system according to the framework of the extended DLVO potential which corresponds to the summation of the electrostatic, van der Waals and magnetic dipole forces. The long-range electrostatic repulsive component is obtained from the Poisson-Boltzmann formalism using the superposition approximation [11] and taking into account for the first time both $\mathrm{pH}$ [12] and the size dependence [10] of the particle surface charge. Treating the nanopaticles as rigid spheres the contribution of van der Waals interactions are calculated without retardation effects. The magnetic interparticle interactions are analyzed applying the dipole model in low coupling regime. In the section 3, a brief description of the chemical synthesis and characterization of the EDL-MF samples investigated and the experimental procedure for the calculation of the nanoparticles surface charge density is revisited. Moreover, after obtaining the $\mathrm{pH}$-dependent phase diagram for the EDL-MF nanoparticles, qualitative observations of the evidenced phases is presented. In section 4 the $\mathrm{pH}$ dependence of the surface charge density is deduced from our analysis so the pair potential interaction is calculated. Finally, the transition lines of the phase diagram are discussed in function of $\mathrm{pH}$ and of nanoparticle mean size.

\section{PAIR POTENTIAL ENERGY FUNCTIONS}

The extended DLVO interparticle potential interaction in EDL-MF is obtained by the superposition of the DLVO contribution (electrostatic repulsion $U_{e}+$ van der Waals $U_{v d w}$ ) with the anisotropic magnetic dipolar $U_{m a g}$ interaction. 
The strongly screened electrostatic repulsions between two identical and charged spheres with radius $R$, separated by a surface-surface distance $D$ can be calculated as [13]:

$$
\frac{U_{e}}{k_{B} T}=\frac{64 k T \varepsilon_{0} \varepsilon_{r} \pi R^{2}}{e^{2}} \tanh \left(\frac{e \Psi_{0}}{4 k T}\right)^{2} \frac{\exp (\kappa D)}{(D+2 R)},
$$

where $\varepsilon_{0}$ is the dielectric constant of the solvent, $e$ the elementary charge, $k_{B}$ the Boltzmann constant, $T$ the temperature, $\Psi_{0}$ the surface potential and $k$ the inverse of the Debye length. Expanding the Poisson-Boltzmann equation up to cubic terms, the surface potential can be calculated as [14]:

$$
\Psi_{0}=\frac{k_{B} T}{2 \bar{B} e} \arcsin h\left(\frac{\sigma_{0} e R 2 \bar{B}}{\varepsilon_{0} \varepsilon_{r} k T(1+\kappa R)}\right),
$$

where the dimensionless coefficient $B$ is dependent on the screening rate $k D$ produced by the electric double layer [14]. The magnitude and the range of the electrostatic repulsive contribution to the interparticle potential are very sensitive to $k$ and $\sigma_{0}$, which were essentially controlled in this work by the adjustment of the ionic strength and $\mathrm{pH}$ of the colloidal dispersions.

Scholten [15] has estimated the van der Waals attraction between iron oxide particles dispersed in aqueous medium, neglecting retardation effects, as follows:

$\frac{U_{v d w}}{k_{B} T}=\frac{A}{6 k_{B} T}\left(\frac{2 R^{2}}{D^{2}+4 R D}+\frac{2 R^{2}}{(D+2 R)^{2}}+\ln \frac{D^{2}+4 R D}{(D+2 R)^{2}}\right)$.

The magnitude of the Hamaker constant $A$ reflects the contribution of the chemical nature of nanoparticles. It depends on both the polarizabilities of the ferrite particles and the dielectric permittivity of the solvent. In the case of EDL-MF particles, $A=1.0 \quad 10{ }^{19} \mathrm{~J}[17]$.

The contribution of the magnetic dipolar interaction can be calculated considering the mean field approximation for low volume fraction regime [17]:

$$
\frac{U_{m a g}}{k_{B} T}=\frac{\gamma^{2}}{48 \pi^{2}} .
$$

The parameter $\gamma$ depends on the interparticle distance and on the individual magnetic moments associated with each particle. In this work $\gamma=0.006$ which characterizes low coupling between particles [16]. This approximation is valid only if $\gamma / 4 \pi<1$ which is in perfect agreement with our results. In this way, the ferrofluid samples can be considered as a dispersion of isolated or independent nanoparticles.

\section{MATERIALS AND METHODS}

\section{III.1. Sample Synthesis and Characterization}

The EDL-MF samples under investigation in this work were prepared using the usual procedure [18]. Briefly, in a first step ferrite nanoparticles were synthesized by hydrothermal coprecipitation of aqueous solutions of $\mathrm{CoCl}_{2}-\mathrm{FeCl}_{3}$ in alkaline medium. By changing both the nature of the base
$\left(\mathrm{NaOH}, \mathrm{CH}_{3} \mathrm{NH}_{2}\right.$ and $\left.\mathrm{NH}_{4} \mathrm{OH}\right)$ and the rate of reagents addition it was possible to obtain nanoparticles of $\mathrm{CoFe}_{2} \mathrm{O}_{4}$ with different mean sizes. In a second step the particles were conveniently peptized in acidic medium by adjustment of the ionic strength, resulting in high quality stable sols labeled as A, B, C and D. The nanoparticles mean sizes were determined by X-rays diffraction experiments from the powder samples, obtained by evaporation of the liquid carrier and were found ranging from 4.5 to $12.0 \mathrm{~nm}$ (Table 1 ).

Table 1 - The corresponding values of the X-rays particles diameter $\left(d_{X R}\right)$, equilibrium constants and the saturation value of the surface charge density for all samples investigated.

\begin{tabular}{c|c|c|c|c}
\hline \hline Sample & $\boldsymbol{d}_{\boldsymbol{X} \boldsymbol{R}}(\mathbf{n m})$ & $\boldsymbol{p} \boldsymbol{K}_{\boldsymbol{I}}$ & $\boldsymbol{p} \boldsymbol{K}_{\boldsymbol{2}}$ & $\mid \boldsymbol{\sigma}_{\boldsymbol{0}}^{\boldsymbol{S A T}}\left(\mathbf{C ~ m}^{\mathbf{2}}\right)$ \\
\hline \hline $\mathrm{A}$ & 4.5 & 4.9 & 9.6 & 0.18 \\
\hline $\mathrm{B}$ & 7.5 & 5.0 & 9.6 & 0.25 \\
\hline $\mathrm{C}$ & 8.8 & 4.9 & 9.6 & 0.26 \\
\hline $\mathrm{D}$ & 11.6 & 5.0 & 9.8 & 0.29 \\
\hline \hline
\end{tabular}

\section{III.2. pH-Dependence of the Surface Charge Density}

It has been evidenced that the EDL-MF nanoparticles become charged when properly dispersed in aqueous media because of the Brönsted acid-base behavior of particles surface [12]. This structural charge is achieved through hydrolysis reactions at surface, which lead to positively charged sites in acidic medium and negatively ones in basic medium. Around neutral $\mathrm{pH}$ most of the surface sites are uncharged. In this way, the $\mathrm{pH}$-dependent chemical equilibrium of protonation/deprotonation can be described by using a two-pK model and the concentration of the charged surface sites can be experimentally determined from potentiometric-conductimetric titrations coupled with the Henderson-Hasselbalch formalism and the mass balance. Then, one can obtain both the saturation value of the structural surface charge density $\left(\sigma_{0}^{S A T}\right)$ and its $\mathrm{pH}$-dependence.

\section{III.3. Potentiometric-Conductimetric Titrations}

In order to determine the concentration of the charged surface sites we have performed simultaneously potentiometricconductimetric acid-base titrations of $40 \mathrm{~mL}$ of each magnetic colloid sample using sodium hydroxide as titrant [12]. The titrations were carried out with an electronic burette Metrohm 751 DOSIMAT. The potentiometric readings were performed with a pHmeter Metrohm 713 using a $\mathrm{pH}$ glass double-junction electrode while the conductivity was measured with a conductometer Metrohm 712 using a conductivity cell specially designed for colloidal dispersions.

\section{III.4. Phase Diagrams}

The $\mathrm{pH}$-dependent phase diagrams for the ferrofluid samples were constructed by using the following procedure. For a volume fraction $\phi=1.8 \%$, many samples of each precursor EDL-MF dispersion at different $\mathrm{pH}(2 \quad p H \quad 7.5)$ and at low ionic strength were prepared in a tube test, $\mathrm{pH}$ being 
adjusted by addition of varying quantities of tetramethylammonium hydroxide (TAMOH). The distinction of the phases was carried out by qualitative experimental observations (Fig. 1). In low $\mathrm{pH}$ values for all samples, the dispersion flowed and did not strongly scatter visible light, even in the presence of magnetic field, evidencing the sol phase (1(a)). Progressive additions of TAMOH increased the viscosity of dispersions because of the partial neutralization of the surface charge. Thus, for samples A, B and C a phase transition was observed where a thixotropic gel could be evidenced. The magnetic colloidal dispersions did not flow when the tube test was tilted (1(b)). Under shaking, the system becomes liquid. Increasing the $\mathrm{pH}$ up to 5.2, all ferrofluid samples coagulated and a macroscopic phase separation with an apparent aqueous supernatant could be evidenced (1(c)).

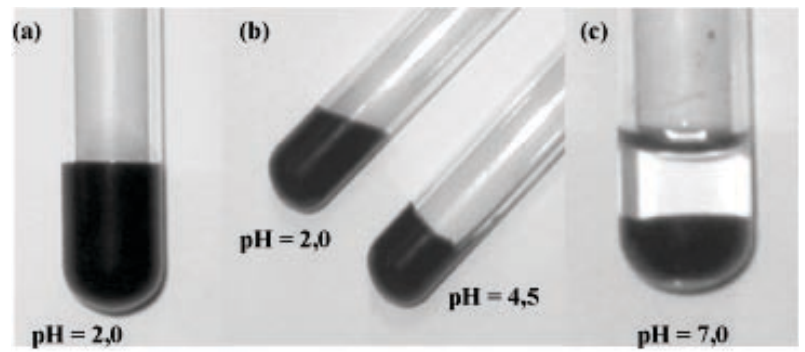

FIG. 1: Qualitative experimental observations of the phase transitions for sample B. (a) Sol phase $(\mathrm{pH}=2.0)$. (b) Comparison between the sol $(\mathrm{pH}=2.0)$ and thixotropic gel $(\mathrm{pH}=4.5)$ phases. Unlike the sol phase, the magnetic colloidal gel does not flow when the tube test is tilted. (c) Fluid-solid transition ( $\mathrm{pH}=7.0)$. A macroscopic phase separation with an apparent aqueous supernatant is clearly evidenced.

Figure 2 presents the observations of the evidenced $\mathrm{pH}$ dependent phases by optical microscopy in the case of sample B. The sol phase (2(a)) is homogeneous on the scale of the optical microscope. In the coagulated state $(2(c))$ it can be seen solid aggregates (dark regions) dispersed in the solvent (clear regions). Macroscopically, the thixotropic gel phase is homogeneous as the sol one. However, observed by optical microscopy (2(b)) it appears as if in an intermediate state, between a sol state and a coagulated one, where some local inhomogeneities are present as in a presumably triphasic system, shown in gray scale. The picture allows to observe a phase diluted in magnetic particles (gray regions), coexisting with a more concentrated one (dark regions). The clear areas refer to the solvent pockets dispersed in the later mentioned phases.

\section{RESULTS AND DISCUSSION}

Figure 3 exhibits a typical simultaneous potentiometric and conductimetric titration result. It shows three distinct regions related to the neutralization of the $\mathrm{H}_{3} \mathrm{O}^{+}$free ions in bulk dispersion, the titration of the surface protons and the excess of base added, respectively. The equivalence points are determined by using the technique of graphical direction lines [19]. Then using the Henderson-Hasselbalch [20] equation, the constants of the chemical equilibrium involved in the surface protonation/deprotonation process $\left(p K_{1}\right.$ and $\left.p K_{2}\right)$ can be
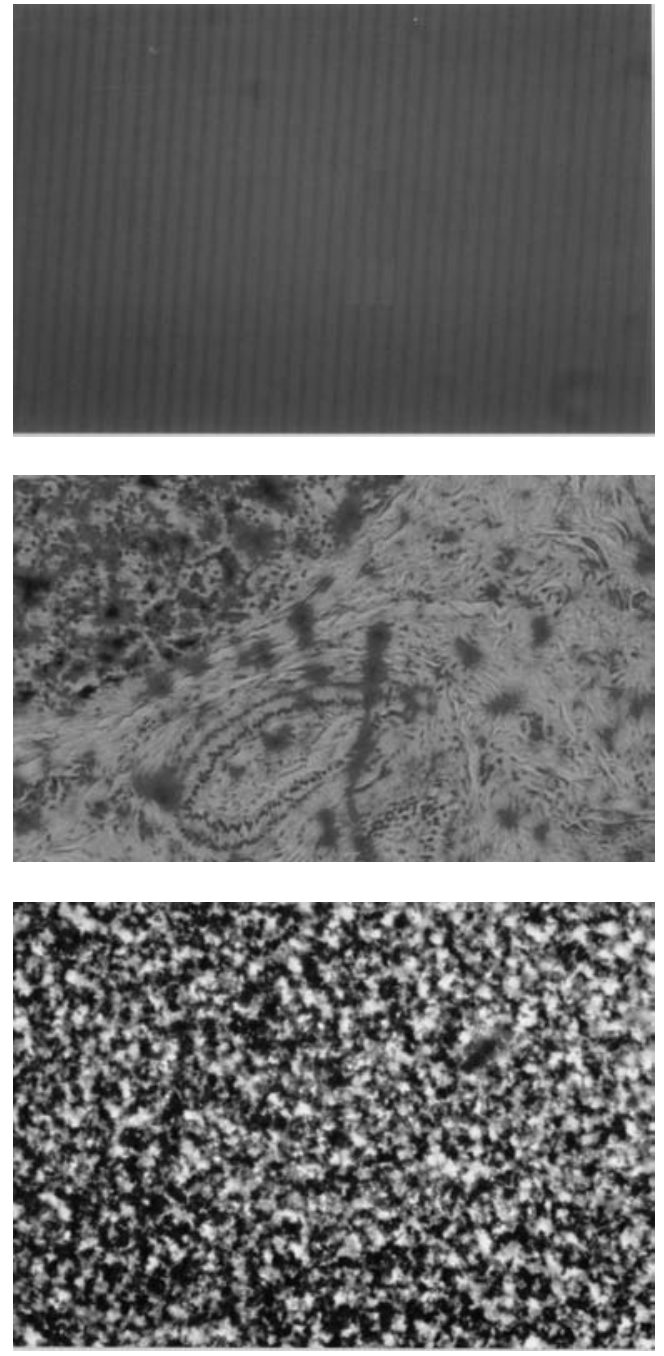

FIG. 2: Microscopic observation pictures of sample B. (a) sol ( $\mathrm{pH}=$ 2.0), (b) gel ( $\mathrm{pH}=4.5)$ and coagulated $(\mathrm{pH}=7.0)$.

obtained. Moreover, the whole superfitial sites concentration $C_{T}$ can be determined using the mass balance. According to the Two-pK model, the surface charge density $\left(\sigma_{0}\right)$ can be calculated as [12]:

$\sigma_{0}(p H)=\frac{F}{A} V\left(\frac{10^{-2 p H}-10^{-\left(p K_{1}+p K_{2}\right)}}{10^{-2 p H}+10^{-\left(p H+p K_{1}\right)}+10^{-\left(p K_{1}+p K_{2}\right)}}\right) C_{T}$,

where $F$ is the Faraday constant, $A$ the total surface area of particles (including the polydispersity) and $V$ the volume of the dispersion. Table 1 summarizes the obtained results of $\sigma_{0}$ for all samples.

Figure 4 shows the $\mathrm{pH}$ dependence of the structural surface charge density for nanoparticles of sample $B$. As it can be seen the surface charge reaches the maximum in extreme $\mathrm{pH}$ conditions where the surface becomes charge saturated. Around the neutral region the nanoparticles charge tends to zero. This $\mathrm{pH}$ dependent profile was evidenced for all samples investigated in this work and it agrees with ultrastable magnetic sols experimentally obtained in acidic or basic mediums. Nevertheless, for $p H<2$ and $p H>12$ the high 


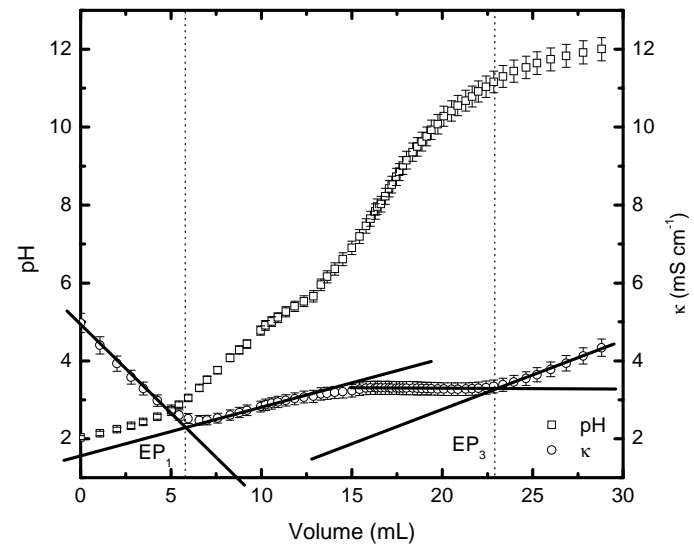

FIG. 3: Typical potentiometric-conductimetric titration curve of EDL-MF samples. EP1 and EP3 are the equivalence points.

ionic strength does not allow stable EDL-MF dispersions, although the particle surface is charge saturated.

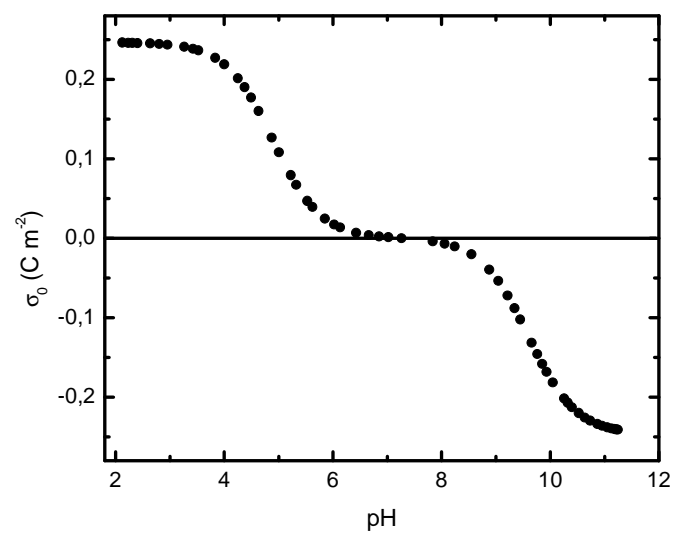

FIG. 4: $\mathrm{pH}$ dependence of the surface charge density for nanoparticles of sample B according to the Two-pK Model. This profile was evidenced for all samples investigated.

The calculated pair potential interaction $U_{T}$ (in $k_{B} T$ units) taking into account the $\mathrm{pH}$ dependence of the surface charge density in the electrostatic repulsion, is shown in figure 5 for some $\mathrm{pH}$ conditions of sample $B$. The potential profile exhibits a primary minimum at short interparticle distances and a positive energy barrier $W$ at short interparticle distances $(D=2 \mathrm{~nm})$. The pair potential may reveal strong long-range repulsion or irreversible aggregation at the primary minimum (coagulation) depending on the height of the energy barrier and on the nanoparticles mean size. In the inset of the figure 5 one can identify a secondary minimum at intermediary interparticle distances $(D=17 \mathrm{~nm})$. If this minimum is moderately deep a reversible aggregation phenomena may occurs (flocculation).

The transition lines of the $\mathrm{pH}$-dependent phase diagram and the height of the energy barrier in function of $\mathrm{pH}$ for sample $B$ are plotted in figure 6. In low $\mathrm{pH}$ medium, the high energy barrier associated to the saturation value of the structural surface charge density ensures the sol phase. The $\mathrm{pH}$ increasing leads to a strong reduction of both energy barrier and nanoparticle charge. Then, a thixotropic gel phase is evi-

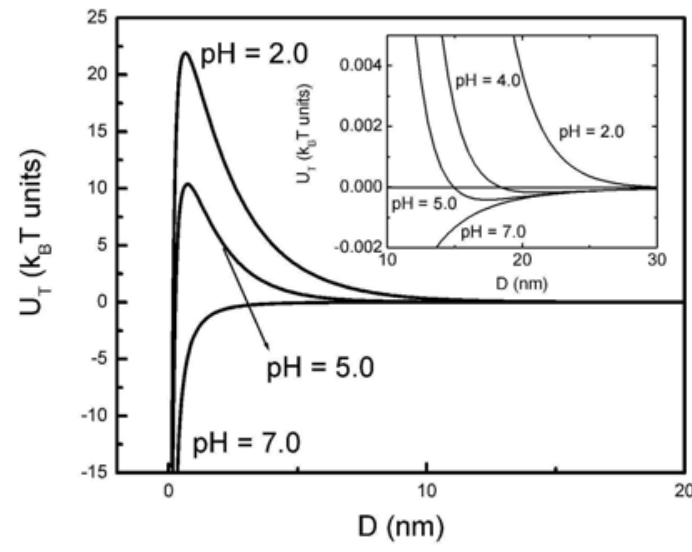

FIG. 5: Pair potential interaction results for some $\mathrm{pH}$ conditions of sample B.

denced in the range of $3.8<p H<5.2$. The existence of this reversible phase transition (flocculation) is clearly related to the decrease of the nanoparticle surface charge about $50 \%$ of its saturation value and to the presence of a secondary minimum whose depth increases for $\mathrm{pH}$ increments in the range of analysis. Simultaneously, the position of this secondary minimum is shifted towards lower values of interparticle distance, evidencing a slight aggregation effect. For $p H \quad 5.2$ in acidic medium, the energy barrier and the surface charge fall drastically so the both minima collapse. Indeed, according to the literature [21] when the energy barrier is lower than $5 k_{B} T$ the thermal fluctuations are sufficient to induce rapid coagulation with a sharp macroscopic fluid-solid phase separation. This phase transition is irreversible due to the strong van der Waals forces which maintain the particles held together and do not break apart without strong external forces.

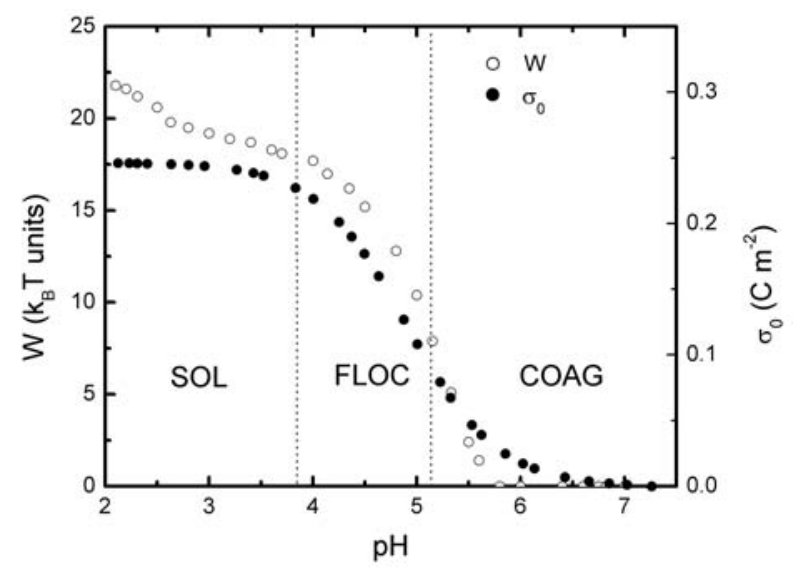

FIG. 6: The pH-dependent phase diagram sample $\mathrm{B}$, the height of the energy barrier (W) and the $\mathrm{pH}$ dependence of the surface charge density. The labels SOL, FLOC and COAG refer to sol, flocculated and coagulated phases, respectively.

Sol-gel thixotropic phase transitions induced by $\mathrm{pH}$ variarions are already well known in many hydrophilic colloidal systems as dispersions of silica nanoparticles. In these systems, where the particle surface presents amphoteric groups, the gelation process results from the formation of hydrogen bonding network between particles [22,23]. As discussed above, 
the sol-gel transition in EDL-MF occurs in the same $\mathrm{pH}$ range where the surface charge strongly decreases compared to its saturation value that implies an increase of the amphoteric surface sites concentration. In this way, these experimental observations are consistent and the thixotropic gel phase in EDL-MF would result from the free energy minimization of the system which would tend to compensate the decreasing of the nanoparticle charge through a surface charge sharing, positive or negative, between particles. This charge sharing suggests a movement of protons by "proton hopping" [24,25] along the hydrogen bonding network of liquid water adjacent to the particles according to the Grotthus mechanism [26,27]. A qualitative and significant fact is that when the nanoparticle surface charge is very small, the system coagulates and the thixotropic gel phase is not evidenced. Moreover, it has been reported that this behavior does not take place in surfacted magnetic fluids $[28,29]$, except under external magnetic field. In this case it has been observed an increase of the system viscosity when the nanoparticles form reversible aggregates [29]. It is worth to underline that in this kind of magnetic fluids the peptization procedure is carried out in non-polar medium, where no charges are involved. Another key factor that enhance our hypothesis is the fact that the strength of hydrogen bonds usually ranges from $4 \mathrm{~kJ} / \mathrm{mol}$ to $20 \mathrm{~kJ} / \mathrm{mol}$. This energy is smaller than a covalent bond energy, but greater than the thermal energy $(2.5 \mathrm{~kJ} / \mathrm{mol}$ at room temperature) [1]. Thus, if the hydrogen bonding is present in the system it dominates the properties of the solvent. In fact, one can observe that under a gentle shaking, which would be sufficient to break the hydrogen bonds, the magnetic colloidal recovers a flowing liquid state in a short time scale but the gel regenerates if the sample is left to stand.

In order to investigate the nanoparticle size dependence in the $\mathrm{pH}$ ranges of the observed phase transitions in EDL-MF, we plotted in figure 7 the $\mathrm{pH}$ dependent phase diagram for all samples. The gray bars estimate the uncertainty of the $\mathrm{pH}$ determination for each phase transition. As it shows, the decrease of nanoparticles mean size leads to a significant increase of the $\mathrm{pH}$ range related to the gel phase. In contrast, for larger particles the sol-gel transition occurs in a very narrow $\mathrm{pH}$ range. In the case of sample $D$, it is not evidenced any gel phase. Regarding the phase transitions (GEL COAG or SOL COAG), the nanoparticle mean size has no influence on the $\mathrm{pH}$ of coagulation. In fact, as shown in figure 8, $p H \quad 5.5$ in acidic medium implies $W \quad 5 k B_{T}$, which induces rapid coagulation whatever the nanoparticle mean size.

On the one hand the X-DLVO approach applied in this work leads to good results related to the general comprehension of the $\mathrm{pH}$-dependent phase diagram of EDL-MF. On the other hand the theory is unable to explain the nanoparticles size dependence on the $\mathrm{pH}$ ranges related to the sol-gel phase transitions in the magnetic colloidal dispersions. The X-DLVO potential used in our investigations only includes the interplay between short-range attractive forces (van der Waals and dipolar magnetic interactions) and long-range repulsive force (electrostatic repulsion interaction). However, in general colloidal systems the presence of non-magnetic and non-DLVO forces, which could be monotonic repulsive, monotonic attractive or oscillatory [1], can strongly modify the profile of the pair

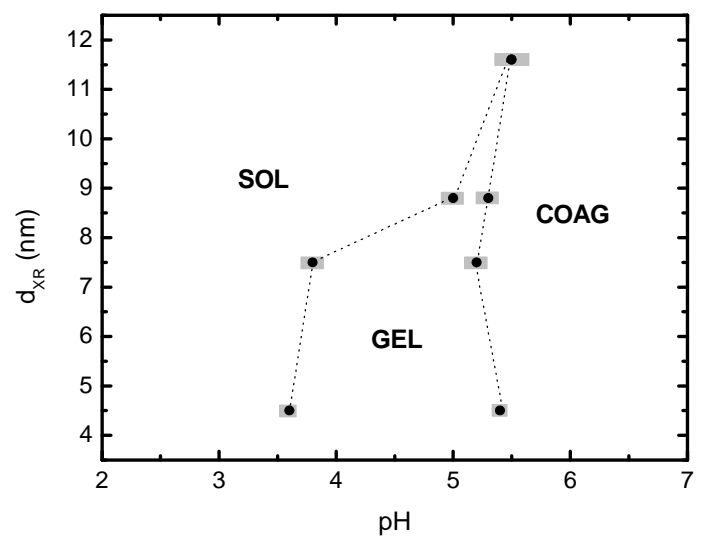

FIG. 7: Nanoparticle size dependence in the $\mathrm{pH}$ ranges of the SOL GEL COAG phase transitions in EDL-MF.

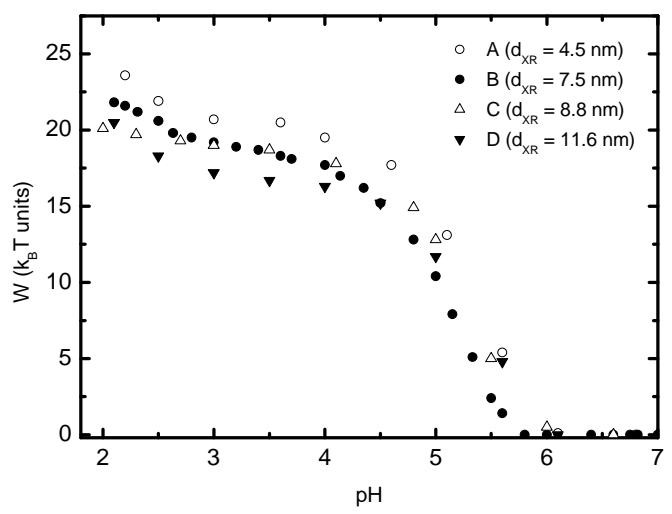

FIG. 8: Height of the energy barrier in function of $\mathrm{pH}$ for all samples investigated.

potential interaction [30]. These additional forces, mainly related to hydration forces (as hydrogen bonding in our case), become preponderant specially as the interparticle distance decreases. Then, concerning the EDL-MF system, the influence of the nanoparticle size in the sol-gel phase transitions requires a more detailed theoretical description of non-DLVO short-range interactions in order to include in the interparticle potential the hydration forces induced by the hydrogen bonding network related to the thixotropic gel phase formation. Despite the influence of hydration forces, the role of the size polydispersity of EDL-MF nanoparticles has to be emphasized. Whatever the preparation method, magnetic colloids always present size distribution. Since the pair potential energy function considers two identical particles, the existence of this polydispersity doubtless affects the diffusion of particles in the solvent, the phase stability of the colloidal system and the interparticle interactions which probably modify the pair potential interaction.

\section{CONCLUDING REMARKS}

This work has focused on the general aspects of colloidal stability of magnetic nanosized particles dispersed in water. 
We have used an extended DLVO potential in order to simulate the interplay between short-range attractive forces and a long-range repulsive force in the system. The profile of the interparticle energy function was successfully employed to investigate quantitatively the phase transitions induced by modifications of the hydronium concentration of the magnetic colloidal dispersions. As a consequence of the Brönsted acidbase behavior of nanoparticle surface, the superficial density of charge proved to be a very important parameter to fine-tune the electrostatic repulsive interparticle potential. In this way, by monitoring experimetally the nanoparticle charge three distinct phases could be evidenced: sol in low $\mathrm{pH}$, coagulated in neutral $\mathrm{pH}$ region and a thixotropic gel phase in $\mathrm{pH}$ ranges between the previous ones, whose existence is related to the formation of a hydrogen bond network in liquid water adjacent to the particles. The transition lines of the con- structed phase diagrams revealed that the increase of nanoparticle mean size implies a significant decrease of $\mathrm{pH}$ ranges related to the gel phase. Nevertheless, the fluid-solid transitions occurred in the same $\mathrm{pH}$ range independing on the particle mean size. In a future attempt we will propose an expression in the pair potential interaction to take into account the nonDLVO forces related to hydration phenomena involved in the gel phase of EDL-MF.

\section{Aknowledgments}

The authors are so grateful to the Brazilian agencies CAPES, CNPq, and FINATEC for their financial support.
[1] J. N. Israelachvili, Intermolecular and Surface Forces, (Academic Press, New York, 1985).

[2] F. Cousin, V. Cabuil, Progr. Colloid Polym. Sci 115, 77 (2000).

[3] F. Cousin, E. Dubois, V. Cabuil Phys. Rev. E 68021405 (2003)

[4] A. Robert, E. Wandersman, E. Dubois, V. Dupuis, R. Perzynski, EuroPhys. Lett. 75, 764 (2006).

[5] E. Hasmonay, A. Bee, J.-C. Bacri, R. Perzynski, J. Phys. Chem. B 103, 6421 (1999).

[6] J.-C. Bacri, R. Perzynski, D. Salin, V. Cabuil, R. Massart, J. Colloid Interface Sci. 132, 43 (1989).

[7] R. Massart, E. Dubois, V. Cabuil, E. Hasmonay, J. Magn. Magn. Mater. Sci. 149, 1 (1995).

[8] O. Sandre, J. Browaeys, R. Perzynski, J.-C. Bacri, V. Cabuil, R.E. Rosensweig, Phys. Rev. E 591736 (1999).

[9] E. Dubois, R. Perzynski, F. Boué, V. Cabuil, Langmuir 165617 (2000).

[10] A. F. C. Campos, F. A. Tourinho, R. Aquino, J. Depeyrot, J. Magn. Magn. Mater. Sci. 310, 2847 (2007).

[11] S. L. Carnie, D. Y. C. Chan, J. Stankovich, J. Colloid Interface Sci. 165, 116 (1993).

[12] A. F. C. Campos, F. A. Tourinho, G. J. da Silva, M. C. F. L. Lara, J. Depeyrot, Eur. Phys. J. E 6, 29 (2001).

[13] W. B. Russel, D. A. Saville, W. R. Schowalter, Colloidal Dispersions, Cambridge University Press, Great Britain, 1989.

[14] A. O. Ivanov, Colloid Journal 59, 446 (1997).

[15] P. C. Scholten, Colloid Chemistry of Magnetic Fluids. Thermomechanics of magnetic fluids; Berkovsky, Hemisphere Pub- lishing Corporation, Bristol, 1978.

[16] C. Menager, L. Belloni, V. Cabuil, M. Dubois, T. GulikKrzywicki, Th. Zemb, Langmuir 12, 3516 (1996).

[17] E. Dubois, V. Cabuil, F. Boué and R. Perzynski, J. Chem. Phys. 111, 7147 (1999).

[18] F. A. Tourinho, R. Franck, R. Massart, J. Mater. Sci. 25, 3249 (1990).

[19] E. Chromiak, Analyst 120, 149 (1995).

[20] D. A. Skoog, D. M. West and F. J. Holer, Fundamentals of Analytical Chemistry (Saunders College Publishing, Orlando, 1996) p 202.

[21] Y. Wang, R. J. Pugh, E. Forssberg, Colloid Surf. A 90, 117 (1994).

[22] T. Jin, D. Finotello, Phys. Rev. Lett. 86, 818 (2001).

[23] L.P. Goodman US Patent 6.617.356 (2003).

[24] K. D. Kreuer, Solid State Ionics 94, 55 (1997).

[25] R. Pomes, B. Roux, Biophys. J 75, 33 (1998).

[26] N. Agmon, Chem. Phys, Lett. 244, 456 (1995).

[27] P. W. Atkins, Physical Chemistry (Oxford University Press, Oxford, 1994).

[28] G. M. Sutariya, R. V. Upadhayay, R. V. Metha, J. of Colloid Interf. Sci. 155, 152 (1993).

[29] S. Odenbach, H. Störk, J. Magn. Magn. Mater. 183, 188 (1998).

[30] D. Grasso, K. Subramaniam, M. Butkus, K. Strevett, J. Bergendahl, Reviews in Enviromental Science \& Biotechnology 1 (2002) 17. 